\title{
Gastroduodenal Fistula: A Rare Finding With an Atypical Presentation
}

\author{
Farah Deshmukh ${ }^{\mathrm{a}, \mathrm{d}}$, Kalpit Devani ${ }^{\mathrm{b}}$, Peter Francisco ${ }^{\mathrm{c}}$, Nancy Merrell $^{\mathrm{c}}$
}

\begin{abstract}
Gastroduodenal fistula (GDF) or double pylorus is a rare, often asymptomatic condition with a prevalence of approximately 0.02 $0.08 \%$. The reported cases have been mainly in Asian countries and more prevalent in males than females. Although the etiology is unclear, Helicobacter pylori and nonsteroidal anti-inflammatory drug use have been associated with the formation of GDF. We present the case of a 65-year-old female with alcoholic cirrhosis and recurrent vomiting who was found to have an antral ulcer. The case includes the serial endoscopic examinations over the period of 7 years and shows the antral ulcer which fistulized into the duodenal bulb creating double pylorus.
\end{abstract}

Keywords: Gastroduodenal fistula; Double pylorus; Pyloric ulcer

\section{Introduction}

Gastroduodenal fistula (GDF) also known as double pylorus is a rare complication of peptic ulcer disease with a prevalence of approximately $0.02-0.08 \%$ and male predominance $[1,2]$. These fistulae can be congenital or acquired [2]. They generally occur in the lesser curvature and connect the gastric antrum with the duodenal bulb [3]. While most of the previously reported cases have been seen in Asian countries, there have been case reports from other parts of the world including Greece and Peru [4-6]. Although Helicobacter pylori $(H$. pylori) and nonsteroidal anti-inflammatory drug (NSAID) use

Manuscript submitted December 11, 2019, accepted January 14, 2020 Published online June 18, 2020

aDepartment of Internal Medicine, Bassett Medical Center and Columbia University College of Physicians and Surgeons, Cooperstown, NY 13326, USA

bivision of Gastroenterology, Department of Internal Medicine, East Tennessee State University, Johnson City, TN, USA

'Department of Gastroenterology, Bassett Medical Center and Columbia University College of Physicians and Surgeons, Cooperstown, NY 13326, USA

${ }^{\mathrm{d}}$ Corresponding Author: Farah Deshmukh, Department of Internal Medicine, Bassett Medical Center and Columbia University College of Physicians and Surgeons, 1 Atwell Rd., Cooperstown, NY 13326, USA.

Email: Farah.Deshmukh@gmail.com

doi: https://doi.org/10.14740/gr1248 have been associated with the formation of the GDF, the actual mechanism of the condition is unknown. The outcome of GDF is variable. In most patients it remains open without any longterm complications while in some cases the two openings can combine to form one opening [7].

GDF is usually an incidental finding and, therefore, endoscopic follow-up of these cases prior to formation of the fistula is very rare [8]. Here, we present the case of a patient who initially presented with a prepyloric ulcer, had serial endoscopic examinations and demonstrated the transformation into GDF.

\section{Case Report}

A 65-year-old female with history of chronic alcohol use was hospitalized for sudden onset of hematemesis. She also reported few days of nausea and vomiting associated with worsening ascites. She denied any significant past medical history except for a ruptured ovarian cyst at age of 30 years that required emergent surgery. Patient had a long history of alcohol use (two glasses of vodka daily) for over 10 years. Physical exam showed scleral icterus, there was mild abdominal distention with epigastric tenderness, no hepatosplenomegaly was noted. No signs of encephalopathy or asterixis were seen. Blood test showed hemoglobin $10.4 \times 10 \mathrm{~g} / \mathrm{dL}$, white blood cell count of $7.7 \times 10^{3}$ cells $/ \mu \mathrm{L}$ and platelet count of $190 \times$ $10^{3}$ cells $/ \mu \mathrm{L}$. Her prothrombin time and international normalized ratio (INR) were elevated at 1.4 and $14.3 \mathrm{~s}$, respectively. Liver function test showed aspartate transaminase $140 \mathrm{U} / \mathrm{L}$, alanine transaminase $29 \mathrm{U} / \mathrm{L}$, alkaline phosphatase $332 \mathrm{U} / \mathrm{L}$, total bilirubin $9.6 \mathrm{mg} / \mathrm{dL}$ (direct $7.4 \mathrm{mg} / \mathrm{dL}$, indirect $2.2 \mathrm{mg}$ / dL). Few hours after admission, she developed an episode of hematemesis with drop in hemoglobin to $7.7 \mathrm{~g} / \mathrm{dL}$. Emergent endoscopy showed a non-bleeding $2 \mathrm{~cm}$ prepyloric gastric antral ulcer (Fig. 1). Biopsy of the ulcer base was negative for malignancy or $H$. pylori.

Follow-up endoscopy at an interval of 6 weeks showed a healing prepyloric antral ulcer with mild portal hypertensive gastropathy (Fig. 2). Patient had a repeat endoscopy at 4 months for persistent nausea and vomiting which again showed a healing antral ulcer. She continued to drink alcohol and was intermittently followed by her primary care physician. She also reported daily NSAID use for abdominal pain and nausea. She had multiple emergency department (ED) visits for the same complaints and underwent repeat endoscopy which 


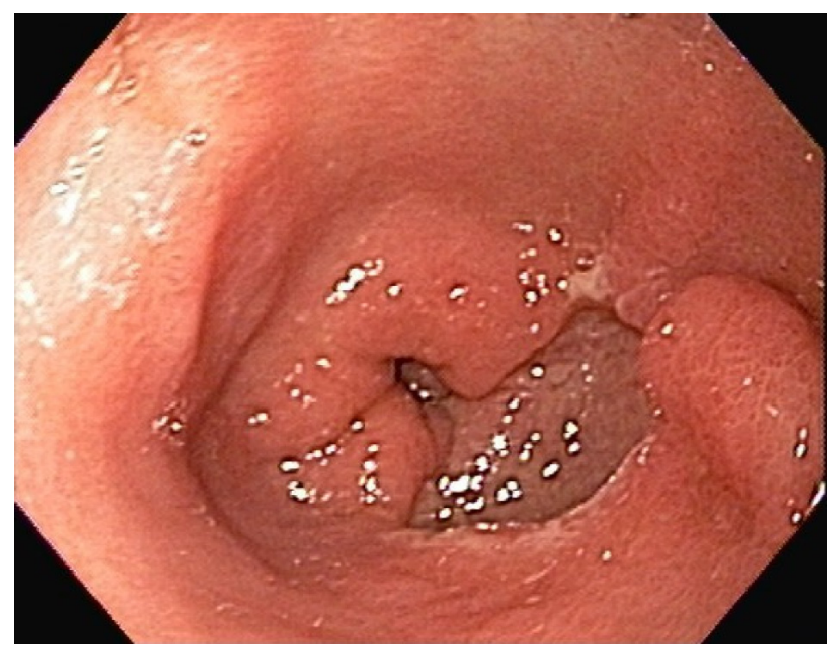

Figure 1. Endoscopic appearance of antral ulcer.

showed a duodenal ulcer (Fig. 3). She was managed with pantoprazole twice daily and was advised to avoid NSAIDs and alcohol. She also underwent extensive workup for recurrent nausea and vomiting which were all unremarkable. Systemic disorders including but not limited to neurological cause, adrenal, parathyroid and diabetes were also ruled out.

She again presented to the ED for worsening nausea and vomiting associated with upper abdominal pain. Endoscopy, now 7 years from the discovery of the initial lesion, showed a GDF. The prepyloric antral ulcer had fistulized into the duodenal bulb creating the appearance of a double pylorus (Fig. 4). Biopsy of the ulcer base was again negative for malignancy or H. pylori. H. pylori stool antigen and serum H. pylori antibody test were also negative. She was discharged on oral proton pump inhibitor and has been followed up with gastroenterology for management of Laennec's cirrhosis. With more frequent outpatient follow up, alcohol abstinence and use of acid-suppression medication, she had gradual improvement in her symptoms in about 1 - 2 months.

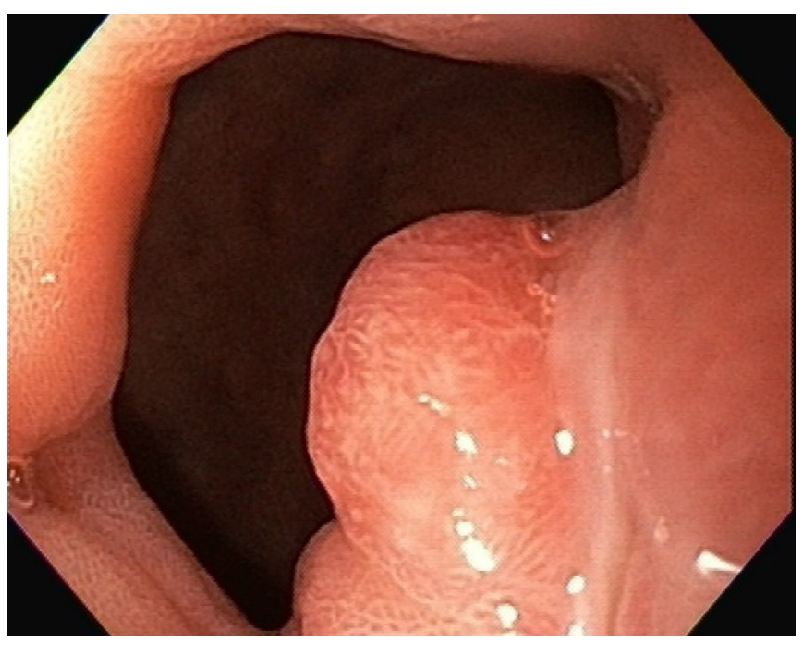

Figure 2. Endoscopic image showing granulation tissue at the site of healing ulcer.

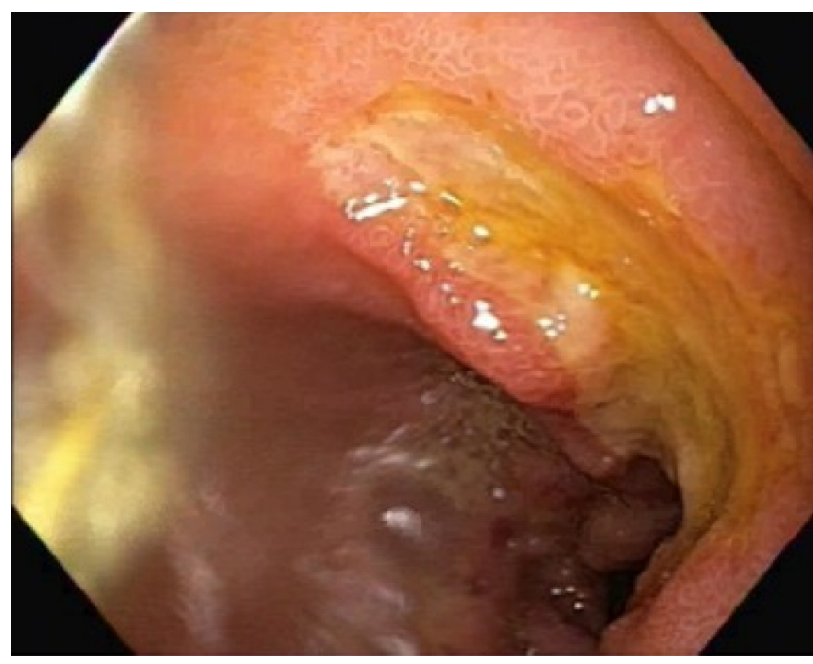

Figure 3. Endoscopic image showing duodenal ulcer.

\section{Discussion}

GDF or double pylorus can be acquired or congenital [9]. When a GDF is identified on endoscopy, the likelihood of congenital origin is more if the patient is young with no history of peptic ulcer disease or other congenital abnormalities [3]. The first cases of GDF have been seen as autopsy findings or incidental findings during surgery [10]. Patients are generally asymptomatic; however, symptoms may range from gastrointestinal bleed to abdominal pain or vomiting [5]. Our patient reported a long history of intractable vomiting associated with abdominal pain. However, due to the history of underlying hepatic cirrhosis it is difficult to identify the actual cause of her symptoms.

The mechanism of formation of GDF is unknown, however, there have been some interesting theories proposed in the past. One of them includes the rare possibility of concomitant duodenal as well as a gastric ulcer that perforated into one another [10]. An alternate theory states that gastric

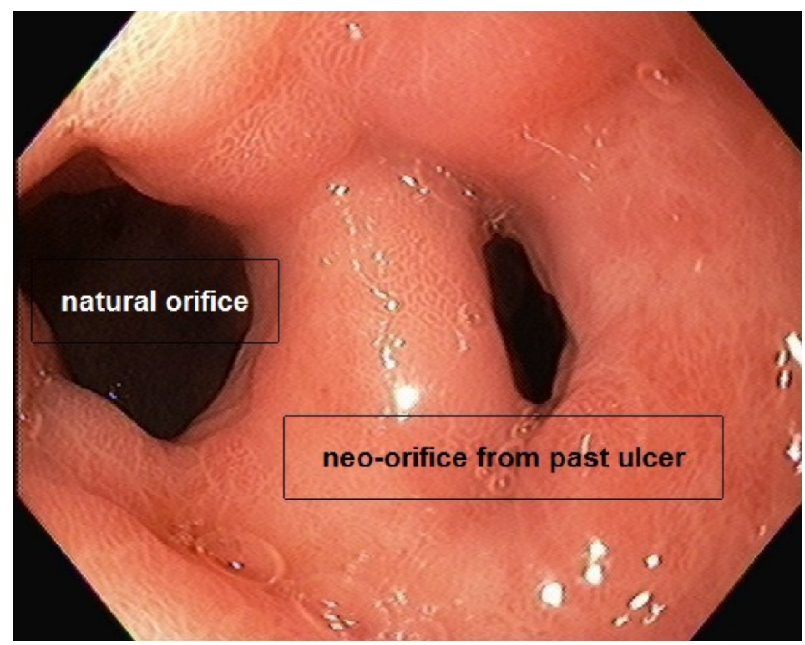

Figure 4. Endoscopic image showing gastroduodenal fistula or double pylorus 7 years from index endoscopy. 
scarring due to gastric ulcer can cause the pylorus to draw up to the lesser curvature with subsequent perforation into the first part of the duodenum [10]. The mechanism of fistula formation in our patient is likely due to fistulization from the gastric antral ulcer during healing. GDF has been associated with systemic diseases such as systemic lupus erythematosus, diabetes, and chronic obstructive pulmonary disease [11]. Medications such as NSAID and steroids have also been associated with interfering with ulcer healing and subsequent development of the fistula $[5,11,12]$. Our patient did not report NSAID use during the initial finding of the prepyloric antral ulcer, however, during her outpatient visits in the following year she reported daily NSAID use for abdominal pain and nausea.

In terms of outcome, GDF remains open in most patients without further complications; however, there have been cases of spontaneous closure reported. Jeon et al reported a case of a 44-year-old Korean male who presented with weight loss and melena, and was found to have a GDF on endoscopy, likely due to previous gastric ulcer [9]. He was treated with proton pump inhibitor and follow-up endoscopy 1 month later showed complete closure of the fistula [9]. There have also been rare cases reported where the two openings have converged to form one large gastroduodenal passage [13]. In terms of management, GDF generally does not require invasive surgical management [14]. Surgery may be indicated if there is no improvement in symptoms with medical therapy or in case of recurrent bleeding, obstruction or perforation [15]. Management primarily involves acid suppression with proton pump inhibitors or histamine $\mathrm{H} 2$ receptor blockers and $H$. $p y$ lori eradication [12]. Additionally, avoidance of NSAIDs and other medications that may impair ulcer healing plays an important part in management [12].

In conclusion, acquired GDF is a rare and often incidental finding during endoscopy. Once identified, it is important to avoid risk factors associated with GDF to avoid complications. Additionally, if there is no improvement with medical management then patients may need surgical intervention. Our case documented serial endoscopies of a patient leading up to the fistula. The likely cause of her GDF was alcohol and NSAID use. She had gradual improvement in her symptoms with alcohol abstinence and use of acid-suppression medication.

\section{Acknowledgments}

None to declare.

\section{Financial Disclosure}

None to declare.

\section{Conflict of Interest}

None to declare.

\section{Informed Consent}

Written informed consent was obtained from the patient for publication of this case report and images.

\section{Author Contributions}

Farah Deshmukh contributed to the drafting and critical revision of the article for important intellectual content, and final approval of the article. Kalpit Devani contributed to the critical revision of the article for important intellectual content, and final approval of the article. Peter Francisco contributed to performing endoscopy, patient treatment and management, and final approval of the article. Nancy Merrell contributed to performing endoscopy, patient treatment and management, and final approval of the article.

\section{Data Availability}

The authors declare that data supporting the findings of this study are available within the article.

\section{References}

1. Yong E, Yong E, Wong JSH, Ho TWT. Acquired double pylorus. Singapore Med J. 2018;59(6):335-336.

2. Umar S, Bilal M. Double pylorus: two sides to one story. Clin Case Rep. 2016;4(12):1211-1212.

3. Chithriki M, Sadiq S, Jaibaji M. Double pylorus. Appl Radiol. 2004.

4. Atiq O, Abrams GA. Case study in gastroenterology \& hepatology: an uncommon complication of peptic ulcer disease. Gastroenterol Hepatol (N Y). 2014;10(5):333334.

5. Fousekis F, Aggeli P, Kotsaftis P, Pappas-Gogos G. Double pylorus: report of a case with endoscopic followup and review of the literature. Gastroenterology Res. 2018;11(2):154-156.

6. Mansur Reimao S, Ferreira De Souza T, Otoch JP, Makoto Sakai C, Yance Hurtado RM, Menezes Marques L, Guimaraes Hourneaux De Moura E, et al. Double pylorus in the era of proton pump inhibitors. Rev Gastroenterol Peru. 2014;34(2):139-140.

7. Hu TH, Tai DI, Changchien CS, Chen TY, Chang WC. Double pylorus: report of a longitudinal follow-up in two refractory cases with underlying diseases. Am J Gastroenterol. 1995;90(5):815-818.

8. Akazawa Y, Mizuta Y, Osabe M, Nakamura T, Morikawa S, Isomoto H, Takeshima F, et al. A case of double pylorus caused by recurrent gastric ulcers: a long-term endoscopic observation. Dig Dis Sci. 2005;50(11):21252128.

9. Jeon BJ, Kim SH, Kim IE, Kim SW, Lee SO, Kim DG, Lee ST. Unusual case of acquired gastrocorporeo-duodenal fistula with long luminal tract treated without surgical 
operation. Journal of Gastroenterology and Hepatology Research. 2013;2:703-705.

10. Moran WH, Jr., Maclean LD. Gastroduodenal fistula; a complication of gastric ulcer. Ann Surg. 1957;146(6):937940.

11. Fattahi MR, Homayoon K, Hamidpour L. Double pylorus in a cirrhotic patient: a case report and review of the literature. Middle East J Dig Dis. 2012;4(2):130-132.

12. Lei JJ, Zhou L, Liu Q, Xu CF. Acquired double pylorus: Clinical and endoscopic characteristics and fouryear follow-up observations. World J Gastroenterol.
2016;22(6):2153-2158.

13. Einhorn RI, Grace ND, Banks PA. The clinical significance and natural history of the double pylorus. Dig Dis Sci. 1984;29(3):213-218.

14. Thompson WM, Norton G, Kelvin FM, Gedgaudas RK, Halvorsen RA, Rice RP. Unusual manifestations of peptic ulcer disease. Radiographics. 1981;1:1-16.

15. Culafic DM, Matejic OD, Dukic VS, Vukcevic MD, Kerkez MD. Spontaneous gastrojejunal fistula is a complication of gastric ulcer. World J Gastroenterol. 2007;13(3):483-485. 